\title{
Long-term adefovir therapy may induce Fanconi syndrome: A report of four cases
}

\author{
FAN PAN $^{1 *}$, YINGCHAO WANG ${ }^{2,3^{*}}$, XIN ZHANG $^{4}$, QINGFENG LIN $^{4}$, \\ XIAOLONG LIU ${ }^{2,3}$, YI JIANG ${ }^{1}$ and CHEN PAN ${ }^{4}$ \\ ${ }^{1}$ Department of Hepatobiliary Surgery, Fuzhou General Hospital, Fuzhou, Fujian 350001; \\ ${ }^{2}$ The Liver Center of Fujian, Fujian Medical University; ${ }^{3}$ The United Innovation of Mengchao Hepatobiliary \\ Technology Key Laboratory of Fujian; ${ }^{4}$ Department of Gastroenterology, Mengchao Hepatobiliary \\ Hospital of Fujian Medical University, Fuzhou, Fujian 350025, P.R. China
}

Received September 6, 2015; Accepted October 21, 2016

DOI: $10.3892 /$ etm.2017.4483

\begin{abstract}
Fanconi syndrome is a rare disease characterized by dysfunction of the proximal renal tubules as a result of various pathogenic events. Drug-induced Fanconi syndrome may be neglected or misdiagnosed, which increases the level of suffering. The aim of the present study was to conduct an investigation into the effects of adefovir (ADV)-induced Fanconi syndrome. Four typical cases of Fanconi syndrome caused by long-term ADV therapy (2-9 years) were diagnosed at our hospital. A complete medical and therapy history was collected from all four patients prior to a physical examination. Laboratory and diagnostic examinations were also conducted. Following this, the patients were diagnosed and a treatment regimen was decided upon. Outcomes of the treatment regimen were observed. Common manifestations of all the four patients were: Renal tubular reabsorption dysfunction; imbalanced electrolyte acid-base ratio; elevated cystatin C levels; severe hypophosphatemia and diffused systemic pain; osteoporosis; and difficultly walking. When ADV was replaced with supportive treatment, all the four patients exhibited
\end{abstract}

Correspondence to: Professor Yi Jiang, Department of Hepatobiliary Surgery, Fuzhou General Hospital, 156 West Erhuan Road, Fuzhou, Fujian 350001, P.R. China

E-mail: jiangyi8183@163.com

Professor Chen Pan, Department of Gastroenterology, Mengchao Hepatobiliary Hospital of Fujian Medical University, 312 Xihong Road, Fuzhou, Fujian 350025, P.R. China

E-mail: panchencry@163.com

${ }^{*}$ Contributed equally

Abbreviations: ADV, adefovir; ETV, entecavir; LMV, lamivudine; MRI, magnetic resonance imaging; PET-CT, positron emission tomography computed tomography; ALP, alkaline phosphatase;

Key words: antiviral therapy, adefovir, Fanconi syndrome, chronic hepatitis B virus, kidney damage, electrolyte disturbances, osteoporosis symptom relief. These findings indicate that long-term ADV therapy may induce Fanconi syndrome. For patients with a history of such therapy, the possibility of Fanconi syndrome should be assessed and monitored closely for indicators, including altered glomerular and renal tubular function. Once diagnosed, the agent should be immediately discontinued and prompt symptomatic treatment should be administered.

\section{Introduction}

Hepatitis B virus (HBV) infection is a considerable health problem worldwide, with an estimated 2 billion of the global population currently infected, of which 250 million are symptomatic (1). Chronic HBV infection in humans may lead to severe liver damage, such as cirrhosis and hepatocellular carcinoma (2). For treatment of chronic HBV, numerous antiviral nucleotide analog drugs have been developed, such as adefovir (ADV), or tenofovir, which are widely used to suppress viral replication as the first-line defence in developing countries (3). $\mathrm{ADV}$ and tenofovir are two nucleoside reverse transcriptase inhibitors; they possess few side-effects, but a number of cases of nephrotoxicity have reportedly been caused by these agents (4-6). A prospective study found that the frequency of renal toxicity was $3-8 \%$ in HBV patients who were treated with ADV therapy for 5 years (7). Thus, this increased number of patients suffering from renal toxicity requires further investigation.

In the present study, four typical cases are presented to investigate this complication. The study protocol was approved by Medical Ethics Committee of Fuzhou Infectious Disease Hospital (Fuzhou, China). All of data were used with the expressed permission of the patients and were published anonymously. Written informed consent was obtained from the patients prior to publication.

\section{Case study}

Case 1. A 61-year-old male patient was referred to our hospital after visiting several hospitals one year ago with pain in both ankles, which gradually expanded to the knees and lumbar back and was accompanied by recent multiple rib fractures. 
Since the onset of these symptoms, the patient had experienced weight loss of $8 \mathrm{~kg}$ and a height decrease of $2 \mathrm{~cm}$. He had a 46-year history of HBV-related hepatitis and a 22-year history of diabetes. Notably, 24 years ago he underwent a splenectomy due to cirrhosis. Since 2008, he had been receiving ADV antiretroviral therapy until admission (5 years in total).

The results of physical examination (data not shown) were predominantly normal, with the exception of the rib cage (which was tender on both the sides), indicating that he was suffering from chronic HBV. In laboratory examination results, $\mathrm{HBs} \mathrm{Ag}, \mathrm{HBeAb}, \mathrm{HBcAb}$ were positive, indicating that the patient was suffering from chronic HBV (Table I).

The observations of the diagnostic examination are recorded as follows:

I: Chest computed tomography (CT): i) Presence of multiple lesions in both lungs, suggesting inflammation; ii) multiple bilateral rib fractures; and iii) reduced pancreas volume with multiple calcification and hardened arteries.

II: Abdominal CT: i) Liver cirrhosis; ii) multiple small cysts in the liver; iii) multiple gallbladder stones; iv) absent spleen; and v) subcutaneous nodules on the right anterior abdomen and spleen injury.

III: Thoracic and lumbar spine magnetic resonance imaging (MRI): i) Thoracic vertebral degeneration; ii) lumbar vertebral degeneration; and iii) protrusion of L3-4, L4-5 intervertebral discs.

IV: MRI of the knees: i) Bilateral contusion in distal femur and proximal tibia indicating a posterior cruciate ligament injury; and ii) bilateral degenerative changes in anterior horn of lateral meniscus and posterior horn of medial meniscus, accompanied by bilateral knee joint effusion and degeneration.

V: PET-CT: i) Multiple lesions on the left lung and right upper lobe were diagnosed of infectious diseases; ii) left maxillary sinusitis; iii) liver cirrhosis, cysts in the right lobe of liver, splenectomy, and portal hypertension changes (enlargement of portal vein and abdominal subcutaneous varicose veins); iv) gall bladder stones, cholecystitis, double kidney small stones and prostate calcification; and v) obsolete fractures on the 3, 4, 6, 7, 9 right ribs and the 6, 7,8 left ribs; and vi) osteoporosis, bilateral sternoclavicular joint degeneration and spinal hyperosteogeny.

VI: Pathological report of renal biopsy: Slight diffusive mesangial proliferation was accompanied by focal/segmental glomerulosclerosis (ischemic sclerosis).

VII: Bone density: Mean bone mineral density (BMD) of the lumbar spine was $0.862 \mathrm{~g} / \mathrm{cm}^{2}$ with a T-score of -3.0 . A dual femur BMD of $0.572 \mathrm{~g} / \mathrm{cm}^{2}$ with a T-score of -3.7 indicated osteoporosis. However, posterior superior iliac spine bone marrow cytological analysis was normal.

The patient was diagnosed with the following complications: i) HBV cirrhosis, compensatory stage; ii) acquired Fanconi syndrome, ADV caused renal tubular lesion; iii) hypophosphatemic osteomalacia; iv) post-splenectomy; and v) type 2 diabetes.
The treatment regimen was as follows: ADV therapy was discontinued after diagnosis and was replaced with entecavir (ETV) antiviral therapy. Meanwhile, the patient received sodium glycerophosphate (2.16 g/day; Sino-Swed Pharmaceutical Co., Ltd., Beijing, China) via intravenous infusion, along with oral administration of calcitriol capsules (0.25 $\mu \mathrm{g}$ /day; Roche Farma, Madrid, Spain) and calcium D3 (1.5 g twice daily; Pfizer, Inc., New York, NY, USA). Following this treatment regimen, body pain was relieved and bone density was improved. The patient was discharged and his treatment was amended to include concentrated divitamins sodium syrup (10 ml, 3 times daily; Liwah Pharmaceutical Co. Ltd., Ningbo, China) for 12 months. Follow-up blood and urine tests were conducted at the indicated time points (Fig. 1), which showed that the alkaline phosphatase (ALP), serum potassium, serum phosphorus, urine acid, urine protein levels returned to normal after 3-month treatment. Urine glucose levels did not return due to the patient's diabetes. His hypophosphatemia was corrected and all body pain was diminished with free movement possible. Following the successful treatment of this patient, our hospital successively admitted another three patients.

Case 2. A 55-year-old male patient visited our outpatient clinic complaining of recurrent fatigue for 16 years with left heel pain having developed in the past six months. A total of 16 years previously, he noted a feeling of malaise and was found to have abnormal liver function (details unknown) and was subsequently diagnosed with HBsAg-positive 'chronic hepatitis' at a local hospital. Since diagnosis, he had been admitted to local hospitals 17 times due to recurrent fatigue and abnormal liver function, including fluctuating alanine aminotransferase and aspartate aminotransferase levels (60-210 U/l and 45-86 U/1, respectively). Diagnosis of HBV liver cirrhosis was confirmed by imaging results and clinical diagnosis. Eight years before admittance to our hospital, the patient was treated with antiviral therapy including lamivudine (LMV), ETV and ADV. Two years prior to admission, the patient's treatment protocol was amended to ETV+ADV antiviral therapy due to recurrent symptoms and the detection of $2.08 \times 10^{6}$ copies $/ \mathrm{ml}$ of $\mathrm{HBV}$ DNA during follow-up. Six months before admittance, the patient began suffering from pain in the left heel, which worsened after movement. Thus, the patient visited our outpatient clinic for further treatment.

The patient's clinical history suggested that he was HBsAg-positive for 22 years. Physical examination (data not shown) demonstrated that his left heel was tender with mild lameness. Laboratory examinations (Table II) indicated that the patient suffered from a renal tubular lesion. Bone density results indicated osteoporosis (lumbar spine T-score, -2.9).

The patient was diagnosed with: i) HBV liver cirrhosis according to his disease history; and ii) acquired Fanconi syndrome due to an ADV-induced renal tubular lesion. Following diagnosis, ADV was discontinued while sodium glycerophosphate was administered to improve hypophosphatemia and calcitriol plus calcium D3 (suppliers as aforementioned) was administered to improve osteoporosis. After a month-long treatment regimen, serum phosphorus levels returned to normal and the patient indicated that the left heel pain had been attenuated. To our regret, this patient did 
Table I. Biochemical examination of Case 1.

\begin{tabular}{|c|c|c|c|c|c|c|c|}
\hline Target in blood & Mount & $\uparrow$ or $\downarrow$ & Reference range & Target in urine & Mount & $\uparrow$ or $\downarrow$ & Reference range \\
\hline TBil & $22 \mu \mathrm{mol} / 1$ & $\uparrow$ & $3-25 \mu \mathrm{mmol} / \mathrm{l}$ & $\alpha 1-\mathrm{MG}$ & $2.89 \mathrm{mg} / \mathrm{dl}$ & $\uparrow$ & $0-1.2 \mathrm{mg} / \mathrm{dl}$ \\
\hline ALP & $175 \mathrm{U} / 1$ & $\uparrow$ & $45-125 \mathrm{U} / 1$ & $\beta 2-\mathrm{MG}$ & $0.976 \mathrm{mg} / \mathrm{dl}$ & $\uparrow$ & $0-0.02 \mathrm{mg} / \mathrm{dl}$ \\
\hline GLU & $7.8 \mathrm{mmol} / \mathrm{l}$ & $\uparrow$ & $3.9-6.1 \mathrm{mmol} / \mathrm{l}$ & $\mathrm{P}$ & $21 \mathrm{mmol} / \mathrm{l}$ & & \\
\hline $\mathrm{P}$ & $0.6 \mathrm{mmol} / 1$ & $\downarrow$ & $0.9-1.62 \mathrm{mmol} / \mathrm{l}$ & $\mathrm{Cl}^{-}$ & $81 \mathrm{mmol} / 1$ & $\downarrow$ & $170-250 \mathrm{mmol} / 24 \mathrm{~h}$ \\
\hline \multirow[t]{6}{*}{$\mathrm{K}^{+}$} & $3.45 \mathrm{mmol} / \mathrm{l}$ & $\downarrow$ & $3.5-5.3 \mathrm{mmol} / \mathrm{l}$ & $\mathrm{K}^{+}$ & $35 \mathrm{mmol} / \mathrm{l}$ & & $25-100 \mathrm{mmol} / 24 \mathrm{~h}$ \\
\hline & & & & $\mathrm{Na}^{+}$ & $114 \mathrm{mmol} / \mathrm{l}$ & $\downarrow$ & $130-260 \mathrm{mmol} / 24 \mathrm{~h}$ \\
\hline & & & & $\mathrm{Ca}^{2+}$ & $5.6 \mathrm{mmol} / \mathrm{l}$ & & $2.5-7.5 \mathrm{mmol} / 24 \mathrm{~h}$ \\
\hline & & & & GFR & $67.08 \mathrm{ml} / \mathrm{min}$ & $\downarrow$ & $80-120 \mathrm{ml} / \mathrm{min}$ \\
\hline & & & & Cystatin C & $1.32 \mathrm{mg} / \mathrm{l}$ & $\uparrow$ & $0-1.03 \mathrm{mg} / \mathrm{l}$ \\
\hline & & & & UN & $7.66 \mathrm{mmol} / \mathrm{l}$ & $\uparrow$ & $2.9-8.2 \mathrm{mmol} / \mathrm{l}$ \\
\hline
\end{tabular}

TBil, total bilirubin; ALP, alkaline phosphatase; GLU, plasma glucose; $\alpha 1-\mathrm{MG}$, a1-microglobulin; $\beta 2-\mathrm{MG}, \beta 2$-microglobulin; GFR, glomerular filtration rate; $\mathrm{UN}$, urea nitrogen; $\uparrow$, upregulated; $\downarrow$, downregulated.

not attend follow-ups at our hospital, thus there is a lack of follow-up results.

Case 3. A 53-year-old male patient was admitted to our outpatient clinic complaining of strengthless lower extremities and difficulty walking for two years, which was particularly aggravated in month prior to admission. Two years prior to admission, the patient experienced lower extremity weakness with no evident causes, but was not diagnosed or treated. For one month prior to presentation at our hospital, he suffered from increased difficulty walking, accompanied by limping and incapability in climbing stairs.

Previous medical history (data not shown) revealed that 10 years ago he suffered from HBV infection, liver lesions and type 2 diabetes. Thereafter, this patient received treatment for 'severe chronic HBV and type 2 diabetes at our hospital.

The therapeutic regimen administered to this patient consisted of liver protection therapy and LMV antiviral therapy for three months, which was discontinued after recovery. The patient became resistant to LMV nine years ago, which was replaced with ADV antiviral therapy until present (9 years in total).

Recent physical examination (data not shown) indicated the patient had a dim complexion and bilateral lower extremity muscle dystrophy with a muscle strength value of 4+. Laboratory examinations (Table III) indicated he was a chronically HBV-infected with imbalanced electrolytes and liver and renal tubular lesion. Meanwhile, accessory examination, including electromyography (EMG), suggested neurogenic damage particularly in both lower extremities. Bone density analysis indicated a left phalanx T-score of -3.63 , a Z-score of -3.09 and a ratio of $68 \%$ to peak bone density, suggesting osteoporosis. Abdominal CT scan indicated: Normal morphology, size, and parenchymal density of the liver; no expansion of intrahepatic bile ducts with evenly distributed intrahepatic vessels; normal size and shape of the gall bladder and the spleen, and no intra-abdominal lymph nodes. On the basis of these findings, the patient was diagnosed with: i) chronic HBV; ii) diabetes type 2; and iii) ADV-induced Fanconi syndrome.
The treatment regimen followed was: ADV was discontinued and replaced with ETV antiviral therapy in combination with other supportive treatment, including compensation of potassium with sustained-release tablets of potassium chloride. Phosphate levels were elevated by administering glycerophosphate sodium injection, and osteoporosis was ameliorated with calcitriol and calcium tablets. The patient was discharged after his symptoms improved. The primary monitoring indexes, including electrolytes and urine glucose levels, returned to normal after one month of treatment (Fig. 1).

Case 4. A 59-year-old female patient, who complained of 'joint pain for two years followed by worsening of complications in 2 months', was admitted to our hospital. She had a 20-year history of liver cirrhosis and 12 years ago she underwent a splenectomy. The patient began receiving ADV antiviral therapy in 2007 (6 years prior to admission).

Physical examination (data not shown) of the patient did not reveal any abnormal symptoms, and the laboratory examination (Table IV) presented imbalanced electrolytes, and liver and renal function abnormality. Whole body PET-CT scan recorded the following: i) No abnormal metabolic imaging development; ii) few obsolete lesions in the back section of the left lung; iii) small stones in the left kidney; iv) osteoporosis; and v) an old fracture of the right ankle. On the basis of these observations, the patient was diagnosed with: i) HBV liver cirrhosis (according to her disease history); ii) ADV-induced Fanconi syndrome; iii) hypophosphatemic osteomalacia; and iv) hypokalemia.

Following diagnosis, ADV was discontinued and was replaced with ETV. After phosphorus, potassium and calcium compensation, the patient's symptoms of joint pain were markedly improved. The majority of the parameters were normal during hospital review (Fig. 1), with the exception of ALP.

\section{Discussion}

Fanconi syndrome is a disease characterized by dysfunction of the proximal renal tubules resulting from various pathogenic events. It was firstly reported by Lignae in 1924 and further 
Table II. Physical and laboratory examination of Case 2.

\begin{tabular}{|c|c|c|c|}
\hline Target in blood & Mount & $\uparrow$ or $\downarrow$ & Reference range \\
\hline TBil & $23.2 \mu \mathrm{mol} / 1$ & & $3-25 \mu \mathrm{mmol} / 1$ \\
\hline $\mathrm{Cr}$ & $125.6 \mu \mathrm{mol} / 1$ & $\uparrow$ & $53-115 \mu \mathrm{mmol} / 1$ \\
\hline Urine albumin & $31.0 \mathrm{mg} / 1$ & $\uparrow$ & $<20 \mathrm{mg} / \mathrm{l}$ \\
\hline Inorganic phosphorus & $0.8 \mathrm{mmol} / \mathrm{l}$ & $\downarrow$ & $0.9-1.62 \mathrm{mmol} / \mathrm{l}$ \\
\hline Potassium & $3.48 \mathrm{mmol} / \mathrm{l}$ & $\downarrow$ & $3.5-5.3 \mathrm{mmol} / 1$ \\
\hline$\beta 2-\mathrm{MG}$ & $9.00 \mathrm{mg} / 1$ & $\uparrow$ & $0-0.65 \mathrm{mg} / \mathrm{l}$ \\
\hline NAG & $52.7 \mathrm{U} / \mathrm{g} . \mathrm{Cr}$ & $\uparrow$ & 3-6 U/g.Cr \\
\hline uRBP & $0.86 \mathrm{mg} / 1$ & $\uparrow$ & $<0.7 \mathrm{mg} / \mathrm{l}$ \\
\hline Lactate & $32.4 \mathrm{mg} / \mathrm{dl}$ & $\uparrow$ & $<22 \mathrm{mg} / \mathrm{dl}$ \\
\hline Cystatin C & $1.48 \mathrm{mg} / \mathrm{l}$ & $\uparrow$ & $0-1.03 \mathrm{mg} / \mathrm{l}$ \\
\hline
\end{tabular}

TBil, total bilirubin; $\beta 2$-MG, $\beta 2$-microglobulin; $\uparrow$, upregulated; $\downarrow$, downregulated; NAG, urine N-acetyl- $\beta$-D-glucosaminidase; uRBP, urine retinol binding protein; $\mathrm{Cr}$, creatinine.

Table III. Biochemical examination of Case 3.

\begin{tabular}{|c|c|c|c|c|c|c|c|}
\hline Target in blood & Mount & $\uparrow$ or $\downarrow$ & Reference range & Target in urine & Mount & $\uparrow$ or $\downarrow$ & Reference range \\
\hline $\mathrm{Cr}$ & $86 \mu \mathrm{mol} / \mathrm{l}$ & & $53-115 \mu \mathrm{mmol} / 1$ & Urine glucose & $3+$ & & - \\
\hline TBil & $12 \mu \mathrm{mol} / \mathrm{l}$ & & $3-25 \mu \mathrm{mmol} / \mathrm{l}$ & $\beta 2-\mathrm{MG}$ & $3179 \mathrm{mg} / \mathrm{l}$ & $\uparrow$ & $<300 \mathrm{mg} / \mathrm{l}$ \\
\hline ALP & $129 \mathrm{U} / 1$ & $\uparrow$ & 45-125 U/1 & & & & \\
\hline BUN & $6.8 \mathrm{mmol} / \mathrm{l}$ & & $2.9-8.2 \mathrm{mmol} / 1$ & & & & \\
\hline $\mathrm{Cl}^{-}$ & 107 mmol/l & & 96-108 mmol/1 & & & & \\
\hline $\mathrm{Ca}^{2+}$ & $2.35 \mathrm{mmol} / \mathrm{l}$ & & $2.08-2.6 \mathrm{mmol} / \mathrm{l}$ & & & & \\
\hline $\mathrm{P}$ & $0.78 \mathrm{mmol} / 1$ & $\downarrow$ & $0.9-1.62 \mathrm{mmol} / \mathrm{l}$ & & & & \\
\hline $\mathrm{K}^{+}$ & $2.93 \mathrm{mmol} / 1$ & $\downarrow$ & $3.5-5.3 \mathrm{mmol} / 1$ & & & & \\
\hline
\end{tabular}

Cr, creatinine; TBil, total bilirubin; ALP, alkaline phosphatase; BUN, blood urea nitrogen; $\beta 2$-MG, $\beta 2$-microglobulin; $\uparrow$, upregulated; $\downarrow$, downregulated.

Table IV. Biochemical examination of Case 4.

\begin{tabular}{|c|c|c|c|c|c|c|c|}
\hline Target in blood & Mount & $\uparrow$ or $\downarrow$ & Reference range & Target in urine & Mount & $\uparrow$ or $\downarrow$ & Reference range \\
\hline ALP & $264 \mathrm{U} / 1$ & $\uparrow$ & 45-125 U/1 & Urine glucose & $1+$ & & - \\
\hline Cystatin C & $1.15 \mathrm{mg} / \mathrm{l}$ & $\uparrow$ & $0-1.03 \mathrm{mg} / \mathrm{l}$ & $\mathrm{pH}$ & 7.0 & $\uparrow$ & $5.5-6.5$ \\
\hline $\mathrm{Cl}^{-}$ & $111 \mathrm{mmol} / \mathrm{l}$ & $\uparrow$ & 96-108 mmol/1 & $\mathrm{Cl}^{-}$ & $75.33 \mathrm{mmol}$ & $\downarrow$ & $170-250 \mathrm{mmol} / 24 \mathrm{~h}$ \\
\hline $\mathrm{Ca}^{2+}$ & $2.07 \mathrm{mmol} / \mathrm{l}$ & $\downarrow$ & $2.08-2.6 \mathrm{mmol} / \mathrm{l}$ & $\mathrm{K}^{+}$ & $31.155 \mathrm{mmol}$ & & $25-100 \mathrm{mmol} / 24 \mathrm{~h}$ \\
\hline $\mathrm{P}$ & $0.48 \mathrm{mmol} / 1$ & $\downarrow$ & $0.9-1.62 \mathrm{mmol} / \mathrm{l}$ & & & & \\
\hline $\mathrm{K}^{+}$ & $2.95 \mathrm{mmol} / \mathrm{l}$ & $\downarrow$ & $3.5-5.3 \mathrm{mmol} / 1$ & & & & \\
\hline
\end{tabular}

ALP, alkaline phosphatase.

defined by Fanconi in 1936 (8). In this syndrome, there is excessive excretion of glucose, bicarbonate, phosphate, uric acid, low molecular weight urinary proteins, potassium, sodium, and calcium, some amino acids, and also water from the urine due to abnormal proximal tubule reabsorption and $1 \alpha$-hydroxylase dysfunction. As a consequence, patients present with clinical features that include phosphate in the urine, renal glycosuria, renal aminoaciduria, proteinuria, and hypophosphatemia, etc (9).

In the present study, common manifestations were observed among all the four patients. Firstly, all the patients were all aged above 50 years and were diagnosed as HBV cirrhosis case with long-term use of ADV (2-9 years). Secondly, the patients all suffered from systemic bone pain, osteoporosis, 

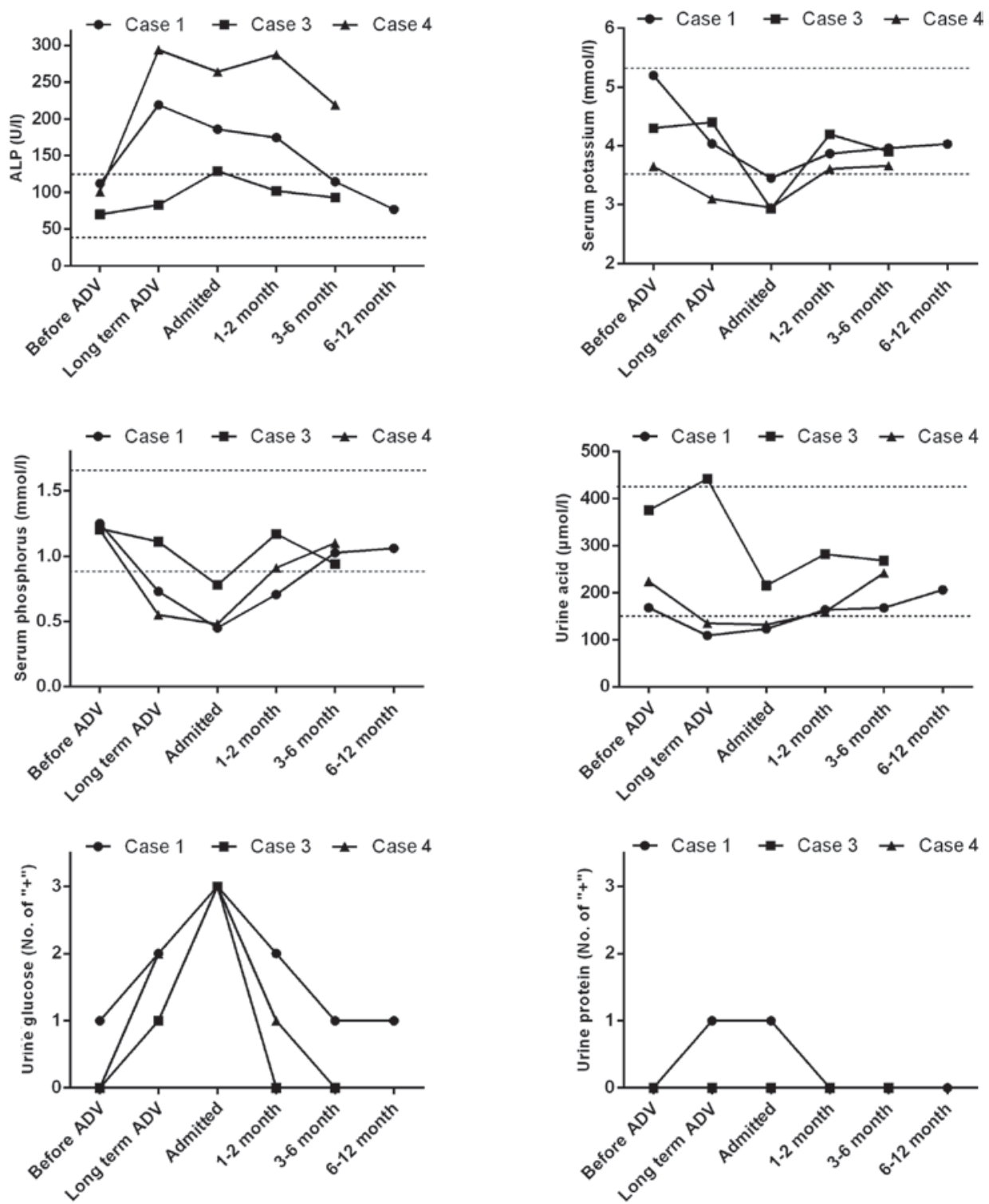

Figure 1. Laboratory examination of three patients before and after hospital admission. On the x-axis, 'Admitted' denotes when the patients received the treatment for Fanconi syndrome; the normal range was limited between two grid lines. For urine glucose and urine protein test, the drug concentration administered to the patients was used to evaluate the level the patients excreted. ALP, alkaline phosphatase; ADV, adefovir.

and walking difficulties. Thirdly, laboratory examinations showed renal tubular reabsorption dysfunction, imbalanced electrolyte acid-base ratio, and elevated cystatin $\mathrm{C}$ levels, and bone density analysis indicated osteoporosis. Finally, pathological analysis of kidney specimen from Case 1 suggested mild diffusive renal mesangial proliferation accompanied with focal/segmental glomerulosclerosis, mild tubular atrophy plus interstitial fibrosis, and atherosclerosis. All the patients presented with symptoms of severe hypophosphatemia, severe diffused systemic pain and osteoporosis, which could be easily misdiagnosed as osteomalacia caused by other metabolic bone diseases leading to excessive loss of urinary phosphate. ADV-induced Fanconi syndrome was the main reason that contributed to increased urinary excretion of phosphorus in all the four cases described in the present study.

Case 1, who was a middle-age man with no family history of genetic disorders or hypoglycemia, exhibited normal serum ceruloplasmin levels. Therefore, this may rule out the possibility of some congenital or inherited genetic disorders, including Pompe's syndrome and amyostatic syndrome. It is more likely for him to have acquired Fanconi syndrome after 5-year therapy of ADV (dose, $10 \mathrm{mg} /$ day), which was administered to treat HBV cirrhosis. Bone marrow biopsy was conducted twice; however, no abnormalities were detected during the examination. In addition, levels of serum globulin and immunoglobulin were normal in plasma of the patient, which may exclude the diagnosis of multiple myeloma and light chain nephropathy. Pathological analysis of renal biopsy showed mild diffusive mesangial proliferation with focal segmental glomerulosclerosis (ischemic sclerosis), mild tubular atrophy plus interstitial fibrosis and atherosclerosis. In recent years, it has been demonstrated that long term use of nucleoside analogues (including ADV and tenofovir) can cause proximal tubule dysfunction namely Fanconi syndrome $(3,10)$. The mechanism that is most likely is that nucleoside analogues may inhibit DNA synthesis in renal 
tubular cell mitochondria resulting in loss of mitochondrial DNA in proximal tubule. In addition, due to their large molecular weights (501 and $636 \mathrm{kDa}$, respectively, according to the Drugbank database; www.drugbank.ca), ADV and tenofovir may cause drug accumulation in the renal tubules, which would disturb the function of transporter proteins, ultimately leading to dysfunction of the proximal tubule $(11,12)$. In recent years, we have observed such patients frequently in clinical practice, in patients who have undergone $>2$ years of ADV therapy (range, 2-9 years) (13); however, the relationship of renal toxicity with drug dose, patient age, and basal kidney diseases requires further research. We hypothesize that the risk factors of kidney damage include age ( $>50$ years), degree of renal impairment before treatment (with or without diabetic nephropathy and hypertensive nephropathy), and drug history (combined with other renal toxic drugs or not). Since two cases (cases 1 and 3) had a history of diabetes, the kidneys of these patients were more susceptible to damage due to the administration of diabetic nephropathy drugs.

Taking into account that the main complaints of the patients were muscle soreness, difficulty walking or spontaneous fractures, many doctors' neglected the detection and diagnosis of Fanconi syndrome induced by drugs and misdiagnosed the patients with multiple myeloma or metastatic tumor of bone from other diseases. Some patients were admitted to rheumatology, orthopedics, nephrology or other departments in different hospitals and still did not receive prompt diagnosis and effective treatment, demonstrating a limited understanding of the disease.

Typical clinical manifestations of hypophosphatemia, such as fatigue, proximal myopathy, dysphagia, intestinal obstruction and systolic dysfunction are not noteworthy and indicative of Fanconi syndrome, unless the phosphorus levels were below $1 \mathrm{mg} / \mathrm{dl}$ (14). The central nervous system may also be involved, causing irritability, delirium or even coma, whereas hemolysis and granulocyte dysfunction may occur rarely $(15,16)$. Chronic hypophosphatemia leads to a decline in bone mineral density, contributing to rickets in children and osteomalacia in adults. In adults, skeletal deformities are relatively rare, although diffusive bone pain may occur all over the body, resulting in weight-bearing ambulation and abnormal gait (17).

The mainstay of treatment of drug-induced Fanconi syndrome is to discontinue the drugs that are suspected to have led to development (including ADV or tenofovir), and switch to an alternative antiviral strategy. Typically, the function of the proximal tubules can be recovered after drug cessation. Meanwhile, electrolyte disturbances and metabolic acidosis due to abnormal proximal tubule reabsorption and excessive loss of bicarbonate, phosphate, potassium, sodium and other substances requires correction. In the present case series, all four patients were diagnosed in a timely manner with prompt discontinuation of ADV in combination with supportive treatment, including calcium compensation and electrolyte imbalance correction. The therapeutic effects were obvious with smooth healing of bone fractures, pain relief and an improvement in walking difficulty noted.

In conclusion, the chances of misdiagnosing or failing to diagnose drug-induced secondary Fanconi syndrome are high. For example, in the present case series $2 / 4$ patients (cases 1 and 3) were diagnosed with Fanconi syndrome after having continuous walking difficulties for 2 years. This increases unnecessary agony and stress for the patients. In clinical practice, Fanconi syndrome should be considered in patients with walking difficulty onset in adulthood accompanied with a history of Fanconi syndrome-inducible medication. Glomerular and tubular function indicators, such as the glomerular filtration rate, serum phosphorus concentration, proteinuria and glycosuria, should be closely monitored when Fanconi syndrome-inducible medication must be administered. Once Fanconi syndrome is diagnosed, the suspected agent should be immediately discontinued with prompt symptomatic treatment.

\section{Acknowledgements}

This work was supported by the National Science and Technology project for the prevention and control of AIDS, viral hepatitis and other major infectious diseases (grant no. 2008ZX10002-005), the National Natural Science Foundation of China (grant no. 81602102), the Natural Science Foundation of Fujian Province (grant nos. 2015J05174 and 2016J01592), the Project of Nanjing Military Region (grant no. 15MS136), the Scientific Research Project of Health and Family Planning Commission of Fujian province (grant nos. 2014-2-43 and 2015-1-94) and the Scientific Foundation of Fuzhou Health Department (grant no. 2014-S-139-3).

\section{References}

1. World Health Organization: Hepatitis B Fact Sheet 204: http://who.int/mediacentre/factsheets/fs204/en/. Accessed January 23, 2013.

2. Zhu H, Wu J and Shen X: Genome-wide association study: New genetic insights into HBV/HCV-related hepatocellular carcinoma genomes. Scand J Gastroenterol: 1-7, 2016.

3. Uteng M, Mahl A, Beckmann N, Piaia A, Ledieu D, Dubost V, Tritto E, Wolf A, Moulin P, Li L, Chibout SD, et al: Comparative renal safety assessment of the hepatitis B drugs, adefovir, tenofovir, telbivudine and entecavir in rats. Toxicol Sci, 2016

4. Verhelst D, Monge M, Meynard JL, Fouqueray B, Mougenot B Girard PM, Ronco P and Rossert J: Fanconi syndrome and renal failure induced by tenofovir: A first case report. Am J Kidney Dis 40: 1331-1333, 2002.

5. Vigano M, Lampertico P and Colombo M: Drug safety evaluation of adefovir in HBV infection. Expert Opin Drug Saf 10: 809-818, 2011.

6. Hadziyannis SJ, Tassopoulos NC, Heathcote EJ, Chang TT, Kitis G, Rizzetto M, Marcellin P, Lim SG, Goodman Z, Ma J, et al: Long-term therapy with adefovir dipivoxil for $\mathrm{HBeAg}$-negative chronic hepatitis B for up to 5 years. Gastroenterology 131: 1743-1751, 2006.

7. Fontana RJ: Side effects of long-term oral antiviral therapy for hepatitis B. Hepatology 49 (Suppl 5): S185-S195, 2009.

8. Mathew G and Knaus SJ: Acquired Fanconi's syndrome associated with tenofovir therapy. J Gen Intern Med 21: C3-C5, 2006.

9. Izzedine H, Launay-Vacher V, Isnard-Bagnis C and Deray G: Drug-induced Fanconi's syndrome. Am J Kidney Dis 41: 292-309, 2003

10. Wang XB, Zhu XC, Huang XY, Ye WJ and Wang LX: Fanconi syndrome due to prolonged use of low-dose adefovir. J Res Med Sci 20: 416-419, 2015.

11. George N, Basu G, Mohapatra A, Zachariah U, Abraham P, Korula A, Varughese S, Jacob CK and Tamilarasi V: Adefovir nephrotoxicity in a renal allograft recipient. Indian J Nephrol 25: $180-183,2015$.

12. Fontana RJ: Side effects of long-term oral antiviral therapy for hepatitis B. Hepatology 49: S185-195, 2009. 
13. Lin Y, Pan F, Wang Y, Chen Z, Lin C, Yao L, Zhang X, Zhou R and Pan C: Adefovir dipivoxil-induced Fanconi syndrome and its predictive factors: A study of 28 cases. Oncol Lett 13: 307-314, 2017.

14. Weisinger JR and Bellorín-Font E: Magnesium and phosphorus. Lancet 352: 391-396, 1998.

15. Takeda E: Calcium pros and cons significance and risk of phosphorus supplementation. The necessity of phosphorus supplementation for hypophosphatemia. Clin Calcium 21: 167-170, 2011 (Article in Japanese).
16. Håglin L: Using phosphate supplementation to reverse hypophosphatemia and phosphate depletion in neurological disease and disturbance. Nutr Neurosci 19: 213-123, 2016.

17. Hamnvik OP, Becker CB, Levy BD and Loscalzo J: Clinical problem-solving. Wasting away. N Engl J Med 370: 959-966, 2014. 